\title{
Alterações oculares associadas ao "bungee jumping": relato de caso
}

\author{
Ocular alterations associated with bungeejumping:case report
}

\author{
José Ricardo Diniz ${ }^{1}$ \\ Tiago Eugêenio Faria e Arantes ${ }^{2}$ \\ Roberta Ventura Urbano ${ }^{3}$ \\ Diana Maria Rodrigues Danda ${ }^{4}$ \\ Denízio Dantas Almeida ${ }^{5}$
}

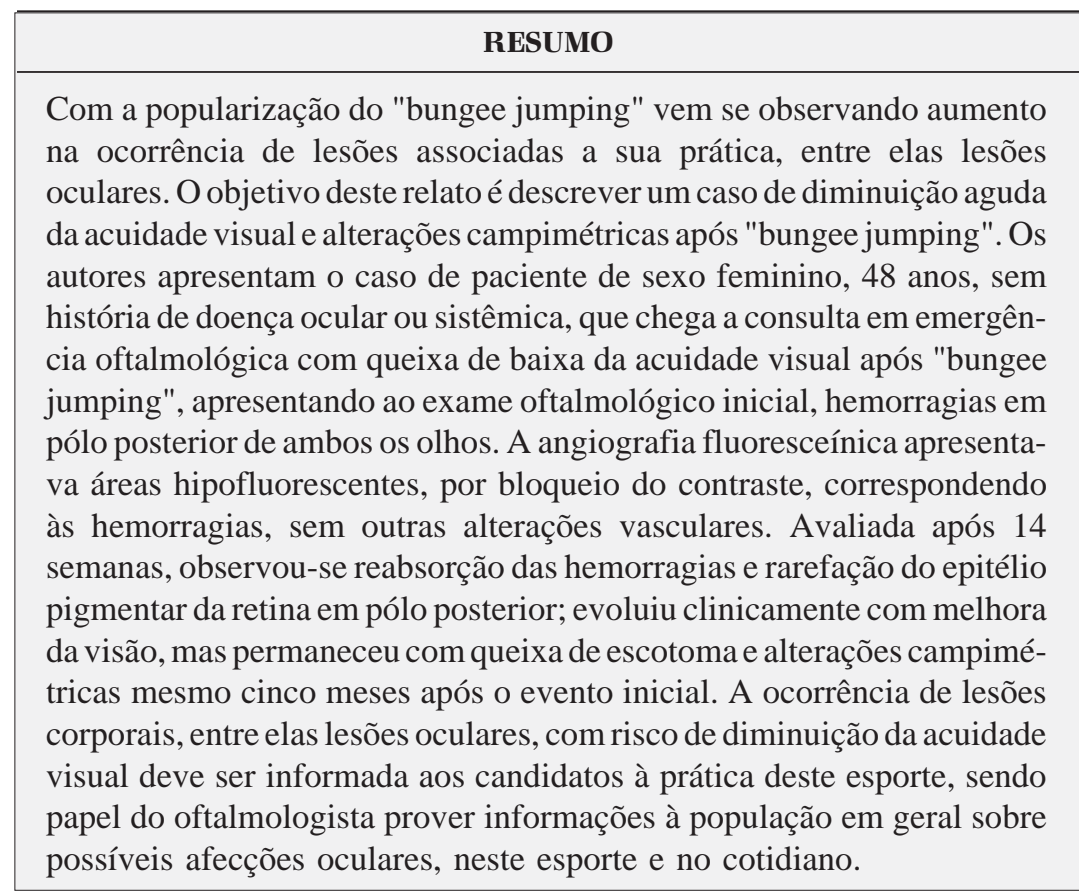

Descritores: Retina/lesões; Traumatismos em atletas; Hemorragia retiniana/etiologia Escotoma/etiologia; Angiofluoresceinografia; Jogos e brinquedos/lesões; Acuidade visual Desaceleração/efeitos adversos; Atividades de laser; Relatos de casos [tipo de publicação]

\section{INTRODUČ̃̃O}

O "bungee jumping" é uma prática originada em ilhas do Pacífico Sul ${ }^{(1-2)}$. Foi introduzido no mundo ocidental no ano de 1955, quando uma equipe da National Geographic aprendeu tal prática com nativos destas ilhas que auxiliavam o exército norte americano nesta área ${ }^{(2)}$. Com a popularização desse esporte vem se observando um aumento do número de casos de lesões associados a sua prática ${ }^{(3)}$.

As lesões de maior gravidade estão relacionadas ao impacto; lesões menores incluem contusões, compressões de extremidades e queimaduras causadas pelo atrito da corda elástica no corpo. Têm sido descritas ainda lesões como hemorragias intra-oculares, enforcamentos e quadriplegias ${ }^{(2)}$.

Há relatos na literatura de casos de lesões oculares associadas à prática deste esporte envolvendo desde hemorragia subconjuntival e quemose a lesões vítreo-retinianas, tais como hemorragias retinianas e pré-retinianas, exsudatos algodonosos, edema macular e hemorragia vítrea, causando redução temporária e, em alguns casos, permanente da acuidade visual ${ }^{(4-7)}$. 
Não sendo encontrado casos descritos na literatura nacional, este relato foi então proposto com o objetivo de descrever um caso de diminuição aguda da acuidade visual e alterações campimétricas após salto de "bungee jumping".

\section{RELATO DE CASO}

Paciente do sexo feminino, 38 anos, branca, professora universitária, hígida, sem antecedentes de doenças oculares ou sistêmicas compareceu ao serviço de urgência oftalmológica do Hospital de Olhos de Pernambuco - HOPE, Recife - PE, com queixa de baixa da acuidade visual e escotoma em ambos os olhos, sendo pior no olho esquerdo. Relatava salto de "bungee jumping" de uma altura de 84 metros, havia três dias.

Ao exame oftalmológico apresentava acuidade visual para longe com melhor correção óptica de 20/20 em olho direito e $20 / 40^{+1}$ em olho esquerdo. Equimose bipalpebral e hemorragia subconjuntival em olho direito, sem outras alterações biomicroscópicas (Figura 1), com pressão intra-ocular equivalente a $6 \mathrm{mmHg}$ em ambos os olhos. Ao exame fundoscópico apresen-

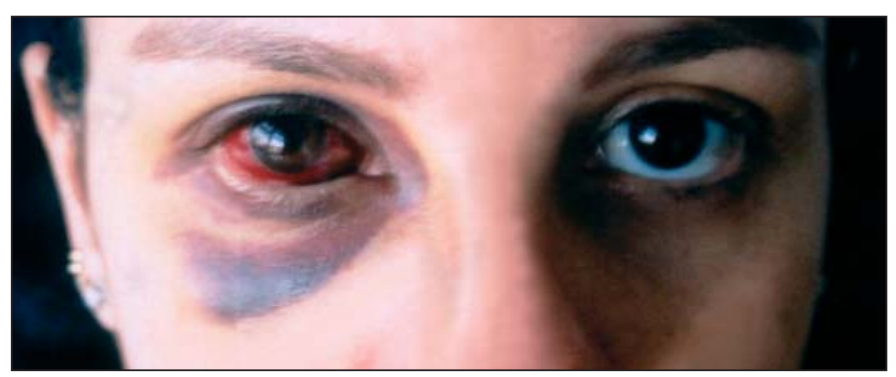

Figura 1 - Equimose bipalpebral e hemorragia subconjuntival em olho direito

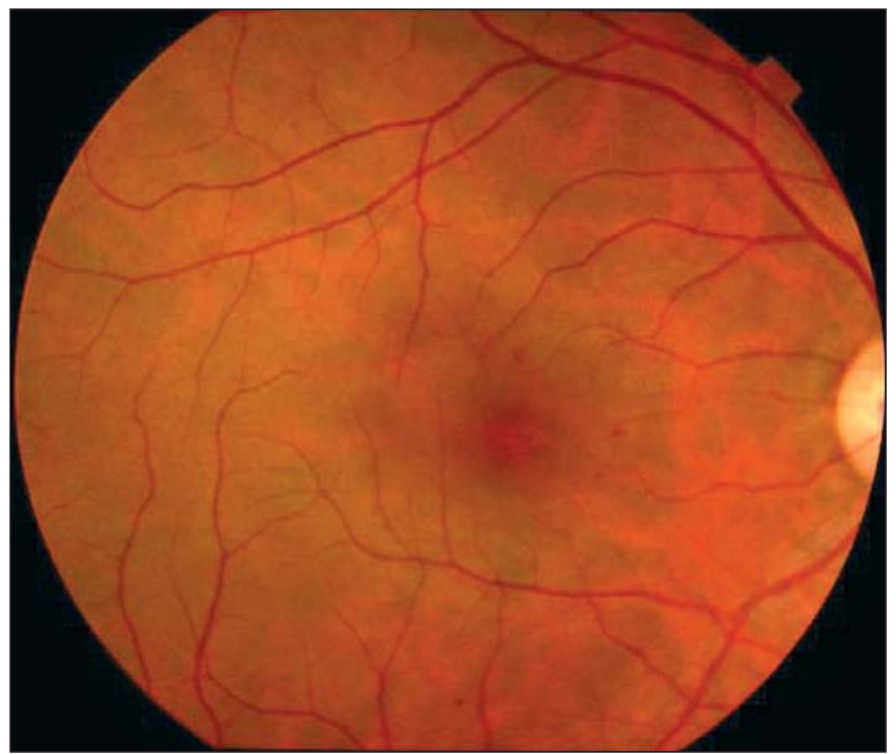

Figura 2 - Retinografia OD - Hemorragias retinianas dispersas no pólo posterior tava disco óptico corado, de bordas nítidas, relação escavação/ disco de 0,3 , retina aplicada e hemorragias retinianas dispersas no pólo posterior, atingindo região macular em ambos os olhos (no olho esquerdo havia uma hemorragia foveal) (Figuras $2 \mathrm{e} 3$ ). Foi submetida à angiografia fluoresceínica, a qual demonstrou em ambos os olhos áreas hipofluorescentes em região macular, por bloqueio do contraste, correspondendo às hemorragias, não se evidenciando sinais de oclusão venosa.

Reavaliada após sete dias, apresentava acuidade visual com correção 20/20 em ambos os olhos, com melhora das alterações de segmento anterior, mas permanecia com queixas de escotoma central. Realizado teste com tela de Amsler evidenciando metamorfopsia em olho esquerdo (Figura 4).

Retornou 14 semanas após consulta inicial ainda com queixa de escotoma central em ambos os olhos. Ao exame apresentava acuidade visual de 20/20, sem alterações ao exame biomicroscópico. À fundoscopia apresentava regressão das hemorragias intra-retinianas, com alterações pigmentares no pólo posterior em ambos os olhos e alteração do reflexo foveal no olho esquerdo.

Cinco meses após a primeira consulta foi realizado campo visual (Central 10-2, Teste limiar, Estratégia: SITA-Standard) com ótimos índices de confiabilidade e limiar foveal preservado $(35 \mathrm{~dB})$. Em olho direito observou-se ausência de sensibilidade em $\pm 3^{\circ}$ do hemicampo temporal e diminuição de sensibilidade em todo hemicampo temporal, mais discreta em hemicampo nasal. Em olho esquerdo detectou-se diminuição da sensibilidade em todo campo central pesquisado e três áreas de ausência de sensibilidade em $\pm 3^{\circ}$ do quadrante temporal superior e inferior (próximo ao meridiano horizontal) e em quadrante nasal (próximo ao meridiano vertical) (Figuras 5 e 6).

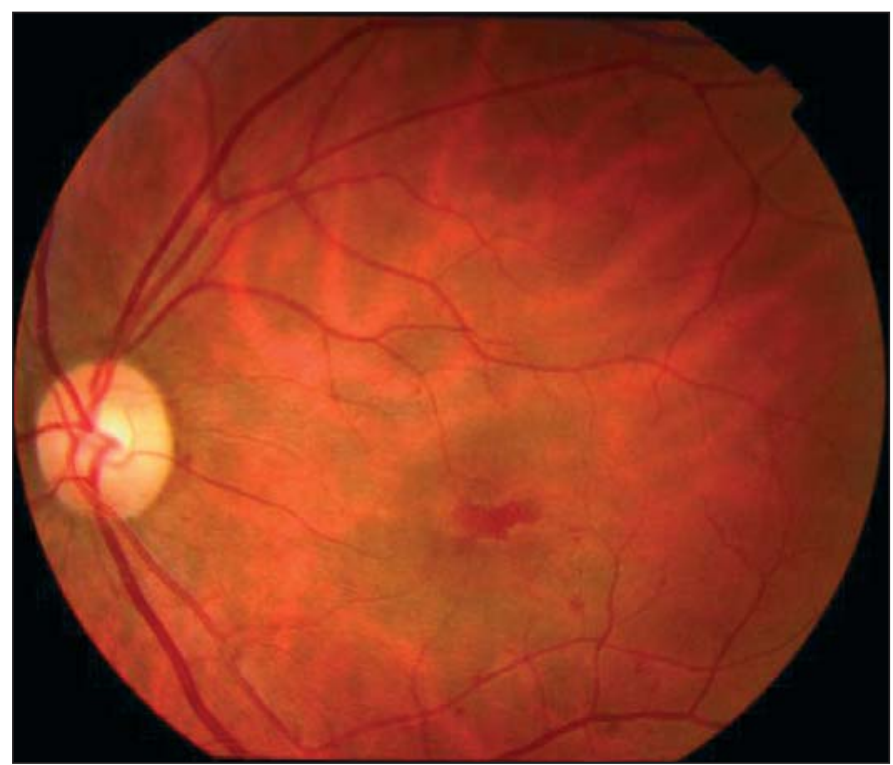

Figura 3 - Retinografia OE - Hemorragias retinianas dispersas no pólo posterior e na região foveal 


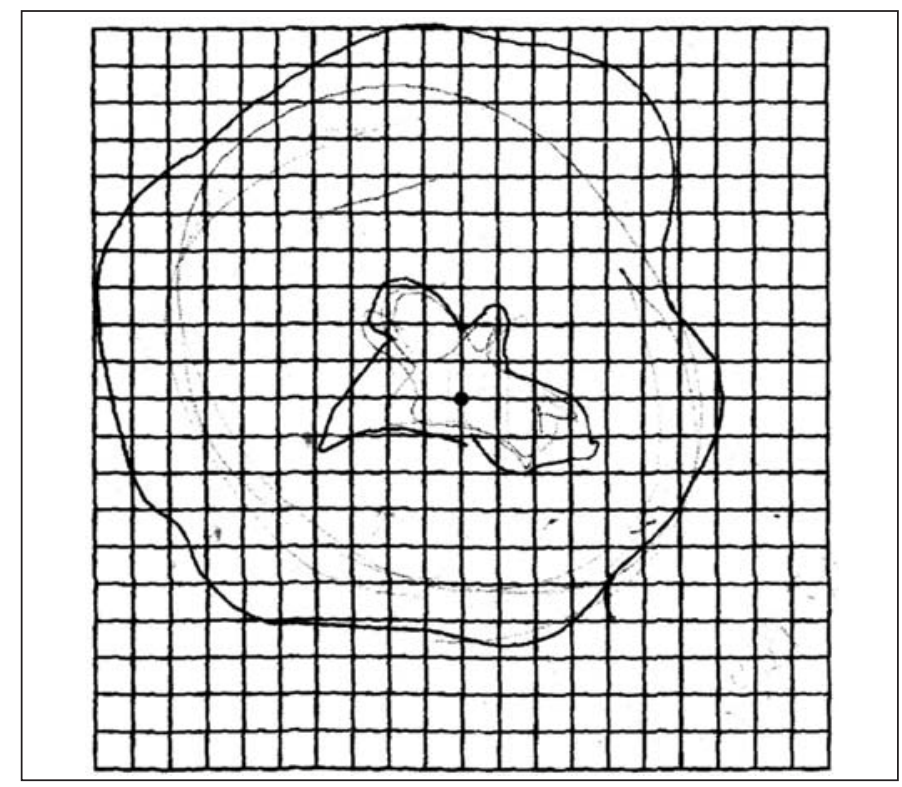

Figura 4 - Tela da Amsler (olho esquerdo) evidenciando distorção da imagem

\section{DISCUSSÃO}

Postula-se que as lesões estejam relacionadas a um rápido aumento da pressão intravascular nas porções superiores do corpo, devido à gravidade, e com a súbita desaceleração que ocorre no final da queda do indivíduo ${ }^{(8)}$. A parte foveolar da retina é extremamente fina e particularmente susceptível ao trauma. Sendo assim, durante o salto, o aumento súbito da pressão intra-torácica causa um aumento repentino da pressão venosa nos olhos e ruptura espontânea dos capilares retinianos superficiais resultando em micro-hemorragias e descolamento hemorrágico da membrana limitante interna na região

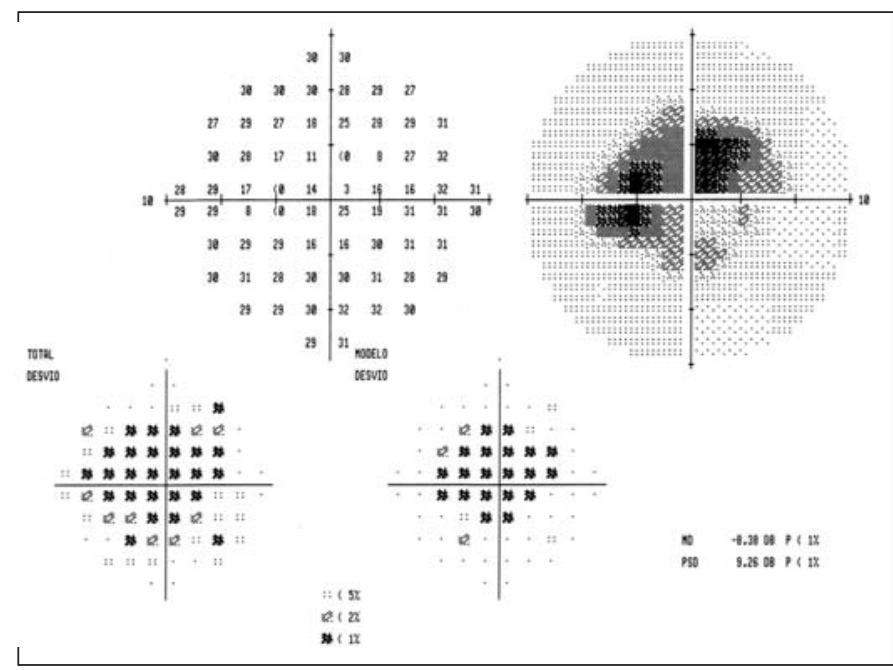

Figura 5 - Campo visual (Central 10-2) OE - Diminuição de sensibilidade no campo central com três áreas de ausência de sensibilidade foveolar $^{(4)}$. O quadro apresenta características semelhantes à retinopatia da manobra de Valsalva e síndrome de Purtscher ${ }^{(9)}$.

No presente caso houve recuperação da acuidade visual, porém a paciente apresentou alterações campimétricas mesmo cinco meses após o evento inicial e permaneceu com queixa de escotoma central. Na literatura há descrição de recuperação visual na maioria dos casos, porém há relato de dois casos onde houve apenas recuperação parcial da acuidade visual ${ }^{(5,8)}$.

A ocorrência de lesões corporais, entre elas lesões oculares, com risco de diminuição da acuidade visual deve ser informada aos candidatos à prática deste esporte. É papel do oftalmologista prover dados epidemiológicos e informações à população em geral sobre possíveis afecções oculares, neste esporte e no cotidiano.

\section{ABSTRACT}

The popularization of bungee jumping is causing an increase in occurrences of lesions associated with its practice, including ocular lesions. The purpose of this study is to describe a case of acute decrease in vision and visual field defects following a bungee jump. The authors present a case of a 48-year-old woman, without history of systemic or ocular disorders, seen at an ophthalmologic emergency service with visual loss complaint following a bungee jump. On initial ophthalmologic evaluation, hemorrhages in the posterior pole of both eyes were found. Fluorescein angiography showed hypofluorescent areas, without other vascular alterations. The patient was evaluated after 14 weeks, the hemorrhages cleared up and there was atrophy of the pigmented epithelium of the retina in the posterior pole, but the patient remained with complaint of scotoma and visual field defects even 5 months after the initial event. The occurrence of body lesions, including ocular lesions, with risk of decrease in visual acuity should be informed to candida-

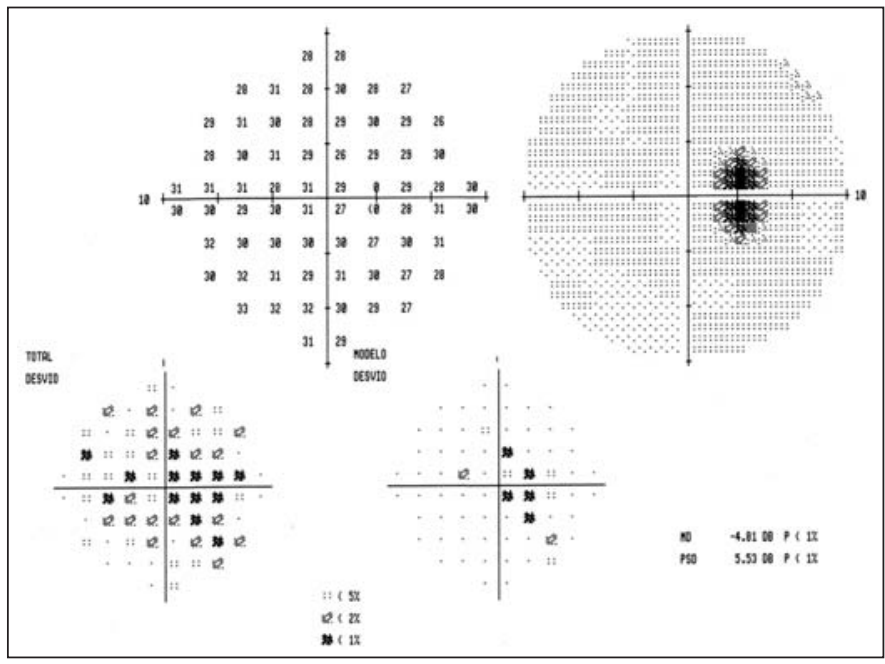

Figura 6 - Campo visual (Central 10-2) OD - Diminuição de sensibilidade com área de ausência de sensibilidade no hemicampo temporal 
tes for the practice of this sport, and it is a for the ophthalmologist to provide information to the population about possible ocular disorders, in this sport and in daily life.

Keywords: Retina/injuries; Athletic injuries; Retinal hemorrhage/etiology; Scotoma/etiology; Fluorescein angiography; Play and playtings/injuries; Visual acuity; Deceleration/adverse effects; Leisure activities, Case reports [publication type]

\section{REFERÊNCIAS}

1. Hite PR, Greene KA, Levy DI, Jackimczyk K. Injuries resulting from bungeecord jumping. Ann Emerg Med. 1993;22(6):1060-3.
2. Vanderford L, Meyers M. Injuries and bungee jumping. Sports Med. 1995;20(6):369-74.

3. Filipe JA, Pinto AM, Rosas V, Castro-Correia J. Retinal complications after bungee jumping. Int Ophthalmol. 1994-95;18(6):359-60.

4. Habib NE, Malik TY. Visual loss from bungee jumping. Lancet. 1994;343 (8895):487.

5. David DB, Mears T, Quinlan MP. Ocular complications associated with bungee jumping. Br J Ophthalmol. 1994;78(3):234-5.

6. Jain BK, Talbot EM. Bungee jumping and intra-ocular haemorrhage. $\mathrm{Br} \mathrm{J}$ Ophthalmol. 1994;78(3):236-7.

7. Innocenti E, Bell TA. Ocular injury resulting from bungee-cord jumping. Eye. 1994;8(Pt 6):710-1.

8. Capão Filipe JA, Rocha-Sousa A, Falcão-Reis F, Castro-Correia J. Modern sports eye injuries. Br J Ophthalmol. 2003;87(11):1336-9.

9. Gomes AMV. Manifestações vítreo-retinianas no trauma ocular contuso. In: Lavinsky J. coordenador. Doenças prevalentes da retina e vítreo. Rio de Janeiro: Cultura Médica; 2002. p.302-5. 